\title{
Equestrian injuries: a five year review of hospital admissions in British Columbia, Canada
}

\author{
Janet M Sorli
}

\begin{abstract}
Aim-To determine the demographics of hospital admissions and mortality associated with equestrian activities in the 33000 riders in British Columbia (BC). Method-Analysis of admission data from the Ministry of Health for the years 199196, review of information obtained from the Office of the Chief Coroner, and comparison of data from Canadian Hospitals Injury Reporting and Prevention Program.

Results-The mean number of admissions per year was 390. Head injury was the most common cause of admission to hospital (20\%) in BC. Females most often required admission (62\%). Teenagers and children have a higher incidence of head injuries than the general population. The injury rate was $0.49 / 1000$ hours of riding. There were three deaths per year, 1/10 000 riders; $60 \%$ were caused by head injury and females predominated.

Conclusion-Head injuries and other serious injuries occur with equestrian activities and it is important for doctors, instructors, and parents to promote the use of appropriate safety equipment, including helmets, especially for children. (Injury Prevention 2000;6:59-61)
\end{abstract}

Keywords: equestrian injuries; horse riding; hospital admissions; helmets

Horse riding is unique in the world of sports because one of the members of the team is not human. The horse has its own athletic abilities and temperament. This athlete weighs upwards of $500 \mathrm{~kg}$, moves at speeds up to $65 \mathrm{kph}$, and elevates the rider up to $3 \mathrm{~m}$ above the ground. The horse can change direction and speed (acceleration or deceleration) in less than a second. When change in direction and speed occur at the same time the centrifugal force is impressive. This team must be in absolute harmony to execute even the simplest maneuvers. The motorcyclist, for example, has a much more predictable partner, although balance and surface conditions are important to both motorcycle and horse riders.

Other significant differences are the teeth and metal shoes of the horse. Both can inflict serious injury to horse handlers on the ground.

1532116 Avenue, Surrey, BC, Canada V4A 1 R6

Correspondence to: Dr Sorli (e-mail jsorli@hotmail.com)
Few statistics are available on the number of equestrian injuries. British Columbia (BC), with 75000 horses, ranks fifth among Cana- dian provinces for horse population and there are an estimated 33000 riders in BC (DVM Olson, Agriculture Canada; personal communication, October 1997). The rate of serious injuries in horseback riding has been reported to be one per 350 to one per 1000 hours of riding $^{2}$ in the USA. This research was undertaken to gain some knowledge of Canadian statistics.

\section{Methods}

Statistics from the Ministry of Health for BC were obtained. The external cause of injury code used was E282.2, which is "accident involving animal being ridden". In $\mathrm{BC}$, the majority of animals being ridden are horses. This also includes fall from, knocked down by, thrown from, trampled by animal being ridden, and ridden animal stumbled and fell. The 0.2 part of the code identifies the injured person as the rider of the animal. The statistics include age, gender, diagnostic code, exit code, length of stay, and hospital of admission.

Diagnoses were grouped according to anatomical location based on the International Classification of Diseases, ninth revision, diagnostic codes. Exit codes were either death, left against medical advice, or discharge from hospital. To capture all deaths, including those dead on arrival, information from the Office of the Chief Coroner, BC Coroner Services, was also obtained. Ages were totaled in groups from $1-5$ years, $6-15$ years, $16-34$ years, and 35 and older. Length of stay was divided into 1 day, 2-6 days, or 7 and more days. Longer stay usually reflects more serious injuries.

\section{Results}

There were 1950 admissions from 1991-96, a mean of 390 per year. During these five years 15 people died, nine of head injuries $(60 \%)$. Sixty per cent of the deceased were female. They varied in age from 4-71 years old. None of those dying of head injury wore a helmet. The $40 \%$ of fatalities who did not die of head trauma were either crushed by the horse, drowned when they fell into rivers, or suffered from multiple trauma, including one cougar and one bear attack on the mounted rider.

The ages of patients admitted ranged from 1-83 years old. Patients under the age of 16 accounted for $25 \%$ of the admissions. Thirty six per cent were 16-34 years old, with the remaining $37 \%$ being $35-83$ years old. Forty two $(2 \%)$ were 5 years old or younger. Forty seven per cent per year stayed only 1 day. Thirty nine per cent stayed 2-6 days, and 14\% stayed between 7 and 
Table 1 Body part injured: $B C$ data $v$ all Canadian (CHIRPP) data (\%)

\begin{tabular}{lll}
\hline Body part & $\begin{array}{l}B C^{\star} \\
(n=1950)\end{array}$ & $\begin{array}{l}\text { CHIRPPt } \\
(n=169)\end{array}$ \\
\hline Head & 20 & 41 \\
Upper limb & 19 & 28 \\
Spine & 7 & NA \\
Trunk & 18 & 11 \\
Lower limb & 18 & 12 \\
Other & 17 & 8
\end{tabular}

${ }^{\star}$ BC ages $1-83$ years.

+CHIRPP 0-19 years.

289 days. Females accounted for $62 \%$ of the admissions to hospital.

The area most likely to be injured was the head $(20 \%)$. The upper limb was injured 19\% of the time. There were equal admission rates for injuries to the lower limb (18\%) and the trunk $(18 \%)$. Spine injuries and spinal cord injuries contributed $7 \%$ of the total. Seventeen per cent were unspecified (table 1).

The most common type of injury was a fracture $(54.2 \%)$. Concussions made up $9 \%$ of the total. The rest were crush injuries, contusions, and unspecified injuries. Many patients had multiple injuries.

The 42 riders less than 5 years of age suffered from a different pattern of injury than their older counterparts. These children had upper limb fractures $48 \%$ of the time, head injury $29 \%$ of the time, and $22 \%$ were lower limb or other injuries. One 4 year old died from a head injury while riding double with her father.

The rate of hospital admissions for equestrians was $11.8 / 1000$ riders or, assuming one hour per day riding on average, $0.49 / 1000$ hours of riding.

\section{Discussion}

The number of hospital admissions per year in $\mathrm{BC}$ and the severity of the injuries, including three deaths per year, indicate that horse riding must be considered to be equivalent to other medium and high impact sports and participants protected appropriately. According to the Canadian Hospitals Injury Reporting and Prevention Program (CHIRRP) data from 1990-

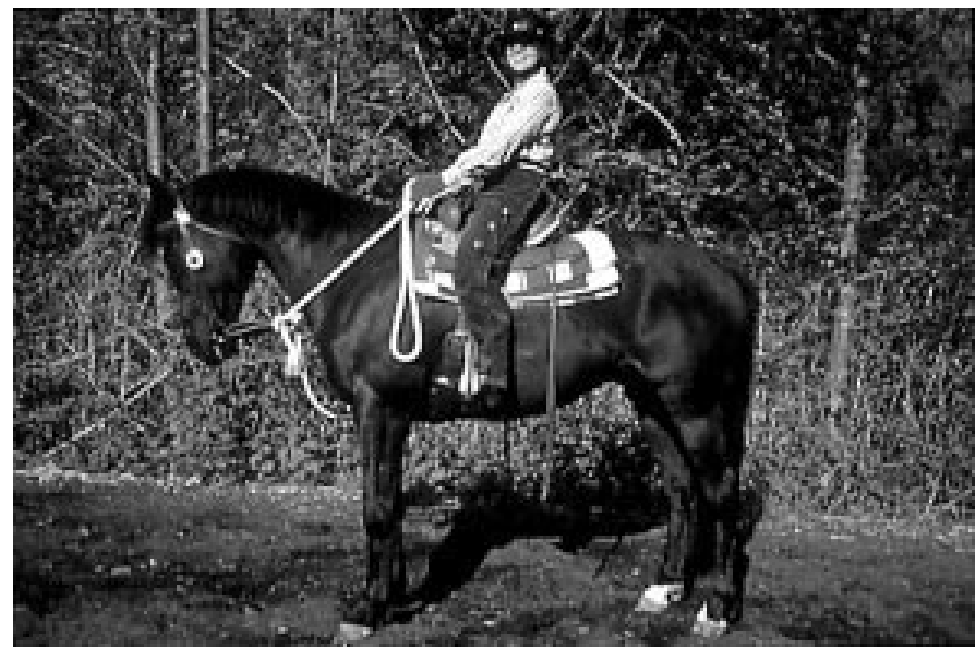

Figure 1 Properly helmeted rider.
$95,14.3 \%$ of injured equestrians presenting to an emergency department require admission. ${ }^{4}$ This compares with a $5 \%$ admission rate for other sources of injury. Therefore, an equestrian athlete, injured sufficiently to present to the emergency department, requires careful examination to rule out serious injury.

Pediatric injuries are of particular concern. The BC five year review included a 1 year old boy who was stepped on by a horse and sustained contusions to his head, neck, and abdomen. Head injuries were more common among toddlers $(29 \%)$ than the general population (20\%). Table 1 compares CHIRPP data, mostly involving 10-14 year olds, with the general BC admissions for riders. It reveals the different injury patterns according to age of the rider. $^{56}$

The rate of serious injuries in horseback riding has been reported to be one per 350 to one per 1000 hours of riding. ${ }^{2}$ The BC study revealed the admission rate to be $0.49 / 1000$ hours riding. Compare this with the injury rate for motorcycle riding, $0.14 / 1000$ hours of riding. ${ }^{2}$ The injury rate requiring emergency services for skiing is 2.91/1000 days, or assuming five hours/day skiing, 0.6/1000 hours. $^{7}$ Since admission to hospital would indicate more serious injury than a visit to the emergency department, we conclude that horse riding is more dangerous than either skiing or motorcycling.

LIMITATIONS OF STUDY

The E code data is not specific as to type of animal being ridden and a number of bull riders do appear in the BC admissions data, although their number is small. The circumstances of the injury are also not available in most instances. The data for admissions also do not include those dead on arrival. The coroner's data and the CHIRPP data are helpful in clarifying these two areas lacking in the $\mathrm{E}$ code data.

\section{Implications for prevention}

A significant reduction in the morbidity and mortality of equestrians could be obtained with the use of American Standard for Testing Materials/Safety Equipment Institute approved helmets by riders (see fig 1). The helmet must be correctly fitted, properly secured, and replaced after significant impact, or every five years. Other safety measures, such as riding boots or breakaway stirrups, correctly chosen mount, certified instructors, and first aid training at horse riding facilities would also be beneficial but no single measure would be as effective as the use of approved helmets for every ride..$^{8-10}$

1 Aronson H. Tough SC horse-related fatalities in the province of Alberta, 1975-1990. Am f Forensic Med Pathol 1993;14:28-30.

2 Bixby-Hammett DM, Brookes WH. Neurologic injuries in equestrian sports. Sports neurology. Aspen: Aspen Publicaequestrian sports. Sports

3 Sorli JM. Equestrian injuries: a survey in British Columbia. American Medical Equestrian Association News 1998; IX(3) May:9-10 
4 Canadian Hospital Injury Reporting and Prevention Program. CHIRPP injury reports. Ottawa: Health Canada, Health Protection Branch, Labo
Control, April 1990-April 1995.

5 Chitnavis JP, Gibbons CLMH, Lloyd Parry J, et al. Accidents with horse: what has changed in 20 years? Injury 1996;27 103-5

6 Nelson DE, Bixby-Hammett DM. Equestrian injuries in children and young adults. Am F Dis Child 1992;146:611-14.

7 Macnab AJ, Cadman R. Demographics of alpine skiing and snowboarding injury: lessons for prevention programs. Inj Prev 1996;2:286-9.
8 American Medical Equestrian Association. Neil Ayer rider safety. (Video.) AMEA, 1997. (Obtainable from AMEA, 4715 Switzer Road, Frankfort, KY 40601, USA.)

9 Ingemarson $\mathrm{H}$, Grevsten S, Thoren L. Lethal horse-riding injuries. F Trauma 1989;29:25-30.

10 Washington State 4-H Club Foundation. Every time...every ride.... (Video.) Washington State 4-H Club Foundation, 1997. (Obtainable from Washington State 4-H Club Foundation, 7612 Pioneer Way, Puyallup, WA 98371-4570, USA.)

\section{Cat flap conundrum}

Here's an odd one, submitted by Dave Chalmers

A real estate agent recently asked if I knew of a standard for cat doors/flaps. Apparently the local council would not certify that a property he was selling complied with the Fencing of Swimming Pools Act, which is the statutory requirement in New Zealand that all domestic swimming pools be fenced. The council's concern was that the cat door, which allowed access from the interior of the house to the pool area for the cat, might also allow access for a small child. The agent also raised the issue of cat doors opening on to other hazardous areas, such as driveways. Does anybody know of a standard for cat doors/flaps, or of any incidents in which children have been injured as a result of venturing into the outside world through the cat door/flap?

PS. Apparently the manufacturers of the cat door in question also manufacture dog doors, on which they have a warning about child access.

If you have an answer, you will find Dave Chalmers at Injury Prevention Research Unit, Department of Preventive and Social Medicine, University of Otago Medical School, PO Box 913, Dunedin, New Zealand, tel: +64 (0) 3479 8510, fax: +64 (0) 3479 8337, e-mail: dchalmers@gandalf.otago.ac.nz.

Side effect of legislation

A little noticed item in a new health bill in Ontario may be an unintended boon to injury prevention. Although the larger purpose of Bill 23 is to give the Minister of Health power to manage hospital restructuring, one clause gives the provincial health insurance plan the right to sue anyone for the costs of health services resulting from negligence or omissions by that person, according to the Globe and Mail newspaper. "If your wife slips on the sidewalk because you failed to shovel, she is unlikely to sue you. Now the government can", the paper concludes. An anonymous senior bureaucrat is quoted as saying that suing people for causing injury was never the legislation's intent and it would not be used for that purpose, but once the law is on the books, who can say how it will be employed by future governments? Perhaps it could be used where other laws have not worked, to improve smoke detector coverage in rental housing, for example. 